01

\title{
Особенности обобщенной синхронизации в системах с запаздыванием
}

\author{
(ㄱ А.Д. Плотникова, О.И. Москаленко
}

Саратовский национальный исследовательский государственный университет им. Н.Г. Чернышевского, Саратов, Россия E-mail: adkoloskova@gmail.com

Поступило в Редакцию 7 марта 2019г.

В окончательной редакции 7 марта 2019 г.

Принято к публикации 15 марта 2019г.

Исследованы особенности режима обобщенной синхронизации в однонаправленно связанных генераторах с запаздыванием. Рассмотрены четыре разных случая взаимодействия систем, характеризующихся различным числом положительных показателей Ляпунова. Установлено, что порог возникновения синхронного режима сильно зависит от степени хаотичности взаимодействующих систем, а связь систем, характеризующихся различным числом положительных показателей Ляпунова, приводит к появлению дополнительных областей синхронизации.

Ключевые слова: системы с запаздыванием, обобщенная синхронизация, спектр показателей Ляпунова, метод вспомогательной системы.

DOI: 10.21883/PJTF.2019.11.47821.17779

Одним из наиболее интересных типов хаотической синхронизации является обобщенная синхронизация, так как этот режим может наблюдаться в двух связанных совершенно разных динамических системах, в том числе и с различной размерностью фазового пространства $[1,2]$. Данный тип синхронизации нашел применение в сфере СВЧ-электроники и радиофизики $[3,4]$, а также в информационно-телекоммуникационных системах для передачи и обработки информации [5-7].

Для исследования синхронных режимов в радиофизических системах одними из самых универсальных и эффективных являются резонансные автогенераторы СВЧ-диапазона с запаздывающей обратной связью на основе многорезонаторных клистронов. Данные приборы отличаются высоким уровнем мощности и КПД, что делает их наиболее перспективными для практического применения. Изучению сложной динамики таких приборов посвящен ряд работ (см., например, [8-10]).

Таким образом, существует необходимость изучения обобщенной хаотической синхронизации в радиофизических системах с запаздыванием. Задачей настоящей работы является исследование особенностей этого режима на примере простой модели двух однонаправленно связанных радиотехнических генераторов с запаздыванием, предложенных в работе [11].

Исследуемая система описывается следующими уравнениями:

$$
\begin{gathered}
\dot{x}_{1}(t)=-x_{1}(t)+k_{1} f\left(x_{1}(t-\tau)\right), \\
\dot{x}_{2}(t)=-x_{2}(t)+k_{2} f\left(x_{2}(t-\tau)\right) \\
+k_{3}\left[f\left(x_{1}(t-\tau)\right)-f\left(x_{2}(t-\tau)\right)\right],
\end{gathered}
$$

где $x_{1}(t)$ и $x_{2}(t)$ - переменные, характеризующие поведение ведущей и ведомой систем соответственно, $k_{1}$ и $k_{2}$ - параметры этих систем, $k_{3}$ - параметр связи, $f(x)=a-x^{2}-$ нелинейная функция, $a=1.5-$ управляющий параметр, время запаздывания $\tau=10$ выбрано одинаковым для обеих систем.

Одним из наиболее распространенных методов детектирования режима обобщенной синхронизации является метод вспомогательной системы [12]. Идея заключается в том, что в бассейне притяжения того же аттрактора, что и у ведомой системы $x_{2}(t)$, вводится идентичная ей вспомогательная система $x_{3}(t)$, но с другими начальными условиями. В случае наступления обобщенной синхронизации после завершения переходного процесса установятся одинаковые функциональные соотношения: $x_{2}(t)=F\left[x_{1}(t)\right]$ и $x_{3}(t)=F\left[x_{1}(t)\right]$. Таким образом, эквивалентность ведомой и вспомогательной систем $x_{2}(t) \equiv x_{3}(t)$ является критерием наличия в системе обобщенной синхронизации.

Для использования описанного метода в дополнение к системе (1) было введено уравнение

$$
\begin{aligned}
& \dot{x}_{3}(t)=-x_{3}(t)+k_{2}^{*} f\left(x_{3}(t-\tau)\right) \\
& +k_{3}\left[f\left(x_{1}(t-\tau)\right)-f\left(x_{3}(t-\tau)\right)\right],
\end{aligned}
$$

где множитель $k_{2}^{*}=k_{2}-10^{-14}$ отвечает за расстройку ведомой и вспомогательной систем, которая вводится для того, чтобы избежать ловушки численного счета.

Системы с запаздыванием характеризуются бесконечномерным фазовым пространством, и соответственно спектр показателей Ляпунова содержит бесконечное число показателей. Следовательно, варьируя параметры (в данном случае $k_{1}$ и $k_{2}$ ), можно наблюдать различные режимы в автономных ведущей и ведомой системах, в частности хаотическую и гиперхаотическую динамику. Таким образом, комбинируя данные величины, можно 


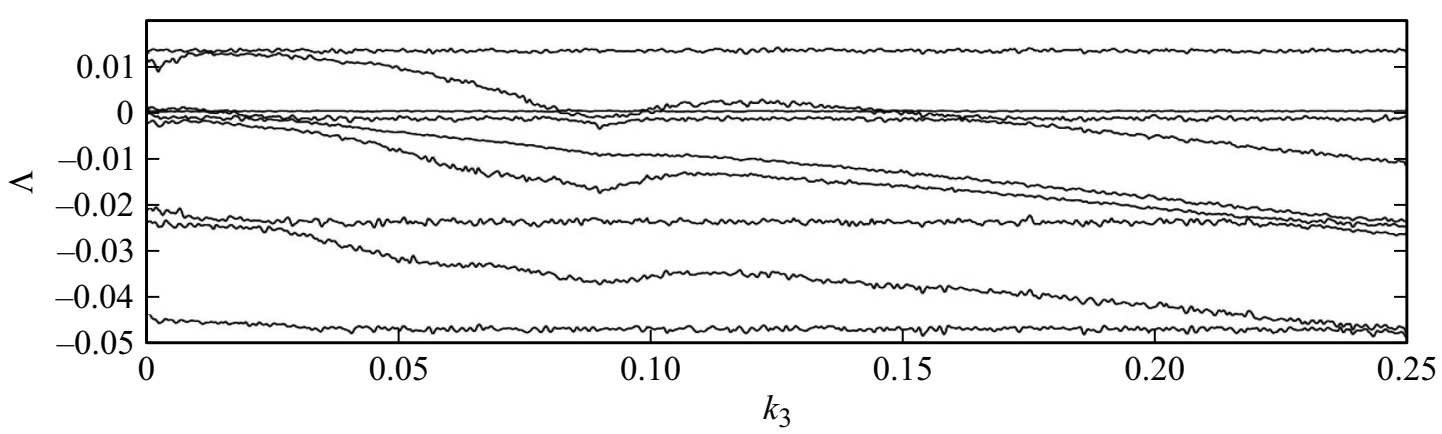

Рис. 1. Зависимости девяти старших показателей Ляпунова от параметра связи $k_{3}$ при $k_{1}=1.005, k_{2}=1.013$ для однонаправленно связанных генераторов с запаздыванием.

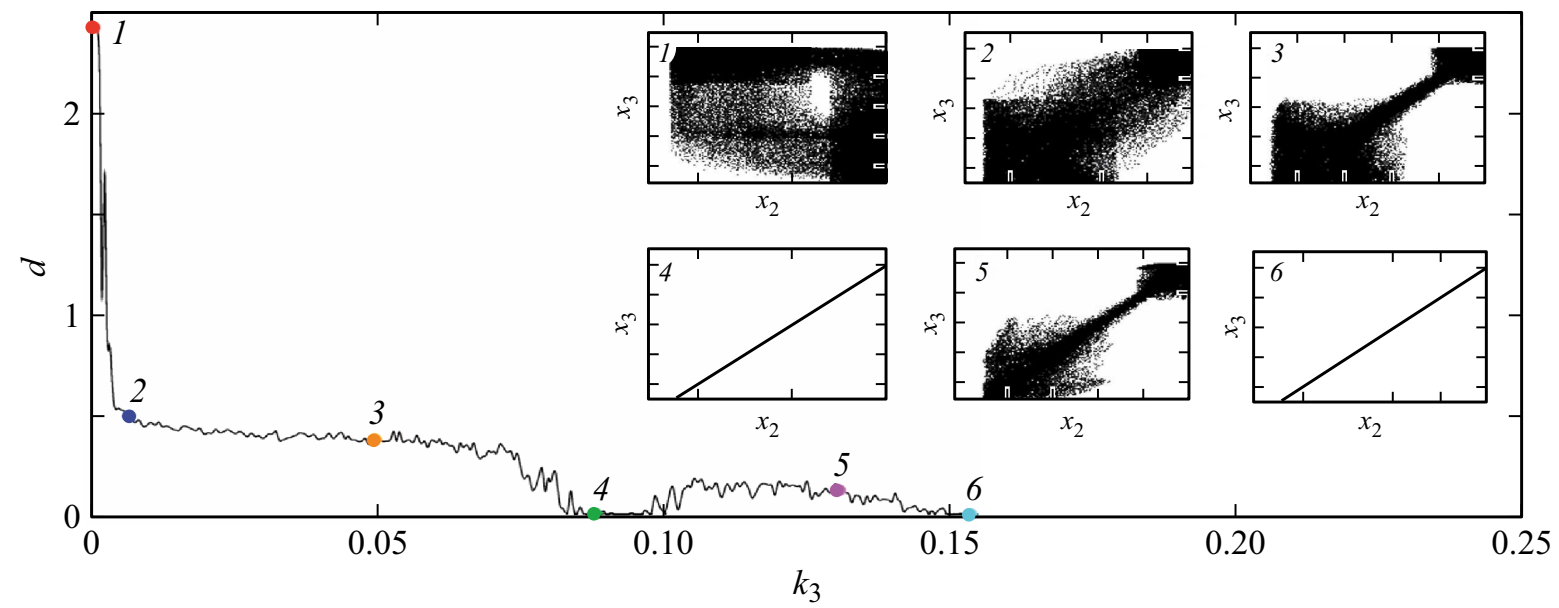

Рис. 2. Зависимость среднего расстояния между состояниями ведомой и вспомогательной систем $d$ от параметра связи $k_{3}$.

исследовать разнообразные варианты влияния ведущей системы на ведомую.

Для расчета спектра показателей Ляпунова однонаправленно связанных генераторов (1) использовался метод, предложенный в работе [13]. Он основан на рассмотрении эволюции во времени пространственного состояния изучаемой системы и (с периодическими ортогонализациями и перенормировками) его возмущений.

Наиболее нетипичная ситуация наблюдается в случае выбора параметров таким образом, что ведущая система в автономном случае характеризуется одним положительным показателем Ляпунова, а ведомая - двумя. На рис. 1 представлен спектр показателей Ляпунова системы (1) для комбинаций параметров $k_{1}=1.005$ и $k_{2}=1.013$.

Из рис. 1 видно, что при увеличении параметра связи $k_{3}$ в системе происходит поочередный переход двух условных положительных показателей Ляпунова в область отрицательных значений, в то время как показатель, отвечающий за ведущую систему, остается неизменным. Следует отметить любопытную закономерность: старший условный показатель Ляпунова переходит в область отрицательных значений дважды, причем первый переход сопровождается последующим выходом этого показателя в положительную область. Когда указанный показатель Ляпунова становится снова отрицательным, в системе окончательно устанавливается режим обобщенной синхронизации.

На рис. 2 представлена зависимость среднего расстояния между состояниями ведомой и вспомогательной систем $d$ от параметра связи $k_{3}$

$$
d=\frac{1}{T-T_{0}} \int_{T_{0}}^{T}\left|x_{2}(t)-x_{3}(t)\right| d t,
$$

где $T$ - время расчета, $T_{0}$ - время переходного процесса. На том же рисунке на вставках приведены плоскости состояний $\left(x_{2}, x_{3}\right)$ в характерных точках по параметру $k_{3}$. Очевидно, что в момент, когда системы становятся эквивалентными друг другу, а величина $d$ обращается в нуль, наступает режим обобщенной синхронизации (плоскости состояний при $k_{3}=0.086$ и 0.156 на рис. 2).

Так же как и на спектре показателей Ляпунова, на зависимости среднего расстояния между состояниями ведомой и вспомогательной систем от управляющего параметра $k_{3}$ (рис. 2) наблюдаются уже две области, где характеристика $d$ обращается в нуль. Несмотря на наличие „нетипичной“ области для режима обобщенной 
Значения порога режима обобщенной синхронизации для различных значений управляющих параметров $k_{1}$ и $k_{2}$

\begin{tabular}{c|c|c|c|c}
\hline$k_{1}$ & Режим & $k_{2}$ & Режим & $k_{3}$ \\
\hline 0.996 & Хаос & 0.988 & Хаос & 0.026 \\
1.013 & Гиперхаос & 1.005 & Хаос & 0.07 \\
& & & & 0.12 \\
1.013 & Гиперхаос & 1.018 & Гиперхаос & 0.142 \\
1.005 & Хаос & 1.013 & Гиперхаос & 0.082 \\
& & & & 0.151
\end{tabular}

синхронизации, на плоскостях состояний наблюдается классический переход к режиму обобщенной синхронизации через перемежаемость (см. вставки на рис. 2). Отчетливо видно, как при увеличении параметра связи $k_{3}$ точки постепенно выстраиваются на главную диагональ, а на границе наступления режима обобщенной синхронизации наблюдается перемежающееся поведение $\left(k_{3}=0.05\right.$ и 0.13$)$.

В целом, установление режима обобщенной синхронизации в системе двух однонаправленно связанных генераторов с запаздыванием происходит по классическому сценарию. В таблице приведены результаты исследования обобщенной синхронизации при нескольких характерных значениях управляющих параметров в порядке увеличения порога установления синхронного режима. Явно видно, что критическое значение параметра $k_{3}$ увеличивается при „усложнении“ динамики системы. Самое малое значение параметра связи $k_{3}$, отвечающего за установление обобщенной синхронизации, имеет место в системе связанных генераторов при таких $k_{1}$ и $k_{2}$, что у ведущей и ведомой систем реализуется хаотический режим в автономном случае. По мере изменения соотношения между значениями управляющих параметров смещается и значение порога обобщенной синхронизации. Отметим, что, когда у обоих генераторов по два положительных показателя Ляпунова, критическое значение параметра $k_{3}$ не является самым большим, как может показаться на первый взгляд. Поскольку в системах присутствует одинаковое количество положительных показателей Ляпунова, на качественном уровне данный процесс будет таким же, как и для первого, самого простого случая. Разница лишь в затрачиваемом „времени“ на установление синхронного режима, откуда и следует больший по величине порог режима обобщенной синхронизации. Наконец, ведущему генератору с одним положительным показателем Ляпунова становится сложнее всего подавить собственную динамику ведомого генератора с двумя положительными показателями Ляпунова, что приводит к наибольшему критическому значению параметра связи.

Таким образом, в работе проведено исследование обобщенной синхронизации в однонаправленно связанных системах с запаздыванием. Для детектирования режима обобщенной синхронизации были использованы метод расчета спектра показателей Ляпунова и метод вспомогательной системы. Показано, что оба метода дают хорошо согласующиеся между собой результаты. В качестве изучаемой модели были выбраны два связанных радиотехнических генератора с запаздыванием с различными управляющими параметрами, отвечающими за разное количество положительных показателей Ляпунова в автономном случае. Показано, что режим обобщенной синхронизации наступает раньше в более простых системах. Наибольшее значение силы связи требуется в случае связанных генераторов, когда ведущая система с хаотическим режимом воздействует на ведомую систему, находящуюся в режиме гиперхаоса.

\section{Финансирование работы}

Работа выполнена при частичной финансовой поддержке Совета по грантам Президента РФ для государственной поддержки молодых российских ученых (проект № МК-531.2018.2).

\section{Конфликт интересов}

Авторы заявляют, что у них нет конфликта интересов.

\section{Список литературы}

[1] Rulkov N.F., Sushchik M.M., Tsimring L.S., Abarbanel H.D.I. // Phys. Rev. E. 1995. V. 51. N 2. P. 980-994. DOI: 10.1103/PhysRevE.51.980

[2] Moskalenko O.I., Koronovskii A.A., Hramov A.E., Boccaletti S. // Phys. Rev. E. 2012. V. 86. N 3. P. 036216. DOI: 10.1103/PhysRevE.86.036216

[3] Стародубов А.В., Короновский А.А., Храмов А.Е., Жарков Ю.Д., Дмитриев Б.С. // Письма в ЖТФ. 2007. Т. 33. B. 14. C. $58-65$.

[4] Kittel A., Parisi J., Pyragas K. // Physica D. 1998. V. 112. N 3-4. P. 459-471. DOI: 10.1016/S0167-2789(97)00186-3

[5] Короновский А.А., Москаленко О.И., Храмов А.Е. // УФН. 2009. T. 179. № 12. С. 1281-1310.

DOI: $10.3367 /$ UFNr.0179.200912c.1281

[6] Terry J.R., VanWiggeren G.D. // Chaos Solitons Fractals. 2001. V. 12. N 1. P. $145-152$.

DOI: 10.1016/S0960-0779(00)00038-2

[7] Пономаренко В.И., Караваев А.С., Глуховская Е.Е., Прохоров М.Д. // Письма в ЖТФ. 2012. Т. 38. В. 1. С. 103-110.

[8] Дмитриев Б.С., Жарков Ю.Д., Рыскин Н.М., Шигаев А.М. // Радиотехника и электроника. 2001. Т. 46. № 5. C. 604-610.

[9] Дмитриев Б.С., Жарков Ю.Д., Клокотов Д.В., Рыскин Н.М. // ЖТФ. 2003. Т. 73. В. 7. С. 105-110.

[10] Shigaev A.M., Dmitriev B.S., Zharkov Yu.D., Ryskin N.M. // IEEE Trans. Electron Dev. 2005. V. 52. N 5. P. 790-797.

[11] Пономаренко В.И., Прохоров М.Д. // Письма в ЖТФ. 2002. T. 28. B. 16. C. 37-44.

[12] Abarbanel H.D.I., Rulkov N.F., Sushchik M. // Phys. Rev. E. 1996. V. 53. N 5. P. $4528-4535$.

DOI: $10.1103 /$ PhysRevE.53.4528

[13] Колоскова А.Д., Москаленко О.И., Короновский А.А. // Письма в ЖТФ. 2018. Т. 44. В. 9. С. 19-25. 